\title{
Analysis of Simulation Parameter of WCDMA for Root Raised Cosine Filter
}

\author{
Qazi Saeed Ahmad ${ }^{1}$, Saif Ahmad ${ }^{2}$, Mohd Javed Khan ${ }^{3}$ \\ Assistant Professor, Department of Electronics \& Communication Engg, Integral University, Lucknow ${ }^{1,2,3}$
}

\begin{abstract}
This Paper basically Represents simulation modal of root raised cosine filter for WCDMA with different parameters of filter. Here we have study the Simulation Parameters (Number of Bits, Bit error rate) of Pulse Shaping FIR Filter at different group delay for WCDMA. W-CDMA is a third-generation (3G) mobile wireless technology that promises much higher data speeds to mobile and portable wireless devices than commonly offered in today's market. It enables several users to transmit their information over the same channel bandwidth. In the mode of transmission a trade off exists between bandwidth containment in frequency domain and ripple attenuation in time domain. It is this trade off of bandwidth containment versus ripple amplitude is considered during system design when developing a data transmission system that employs pulse shaping.
\end{abstract}

Keywords: WCDMA, RRC Filter, DSSS, BER, QPSK, SNR.

\section{INTRODUCTION}

W-CDMA (known as IMT-2000 direct spread spectrum) is a third-generation $(3 \mathrm{G})$ mobile wireless technology that promises much higher data speeds to mobile and portable wireless devices than commonly offered in today's market. W-CDMA can support mobile/portable voice, images, data, and video communications at up to 2 Mbps (local area access) or $384 \mathrm{Kbps}$ (wide area access). It enables several users to transmit their information over the same channel bandwidth. In the mode of transmission a trade off exists between bandwidth containment in frequency domain and ripple attenuation in time domain. It is this trade off of bandwidth containment versus ripple amplitude is considered during system design when developing a data transmission system that employs pulse shaping.

\section{WHAT IS WCDMA?}

Wideband CDMA is a third-generation (3G) wireless standard which utilizes one $5 \mathrm{MHz}$ channel for both voice and data, initially offering data speeds up to $384 \mathrm{Kbps}$. WCDMA was the 3G technology used in the US by AT\&T and T-Mobile.

Wideband CDMA is a third-generation (3G) wireless standard which utilizes one $5 \mathrm{MHz}$ channel for both voice and data, initially offering data speeds up to $384 \mathrm{Kbps}$. WCDMA was the $3 \mathrm{G}$ technology used in the US by AT\&T and T-Mobile.

\section{III.WCDMA CONCEPTS}

The application of signal processing techniques to wireless communications is an emerging area that has recently achieved dramatic improvement in results and holds the potential for even greater results in the future as an increasing number of researchers from the signal process and communications areas participate in this expanding field.
From an industrial viewpoint also, the advanced signal processing technology cannot only dramatically increase the wireless system capacity but can also improve the communication quality including the reduction of all types of interference. The present paper deals with simulation model of square root raised cosine pulse shaping filter for WCDMA with different parameters of the filter at 5Mhz.The present paper deals with study of Simulation Parameters (Number of Bits, Number of Errors) of Pulse Shaping FIR Filter at different group delay for WCDMA. Digital Signal processing techniques are being used to improve the performance of $3 \mathrm{G}$ systems. WCDMA (Wideband Code-Division Multiple Access), an ITU standard derived from Code-Division Multiple Access (CDMA), is officially known as IMT-2000 direct spread spectrum. W-CDMA is a third-generation (3G) mobile wireless technology that promises much higher data speeds to mobile and portable wireless devices than commonly offered in today's market. W-CDMA can support mobile/portable voice, images, data, and video communications at up to 2 Mbps (local area access) or 384 Kbps (wide area access).Wideband Code-division multiple access is one of several methods of multiplexing wireless users. In CDMA, users are multiplexed by distinct codes rather than by orthogonal frequency bands, as in frequency-division multiple access. The enhancement in performance is obtained from a Direct Sequence Spread Spectrum (DSSS) signal through the processing gain and the coding gain can be used to enable many DSSS signals to occupy the same channel bandwidth, provided that each signal has its own pseudorandom (signature) sequence $[4$, $8,18,20,32]$. Thus enable several users to transmit their information over the same channel bandwidth. This is the main concept of a WCDMA communication system. The signal detection is accomplished at the receiver side by knowing the code sequence or signature of the desired user. Since the bandwidth of the code signal is chosen to be much larger than the bandwidth of the information- 
bearing signal, the encoding process enlarges or spreads the spectrum of the signal. Therefore, it is also known as spread spectrum modulation. The resulting signal is also called a spread-spectrum signal, and CDMA is often denoted as spread-spectrum multiple access. A tradeoff exists between bandwidth containment in frequency domain and ripple attenuation in time domain. It is this tradeoff of bandwidth containment versus ripple amplitude which must be considered by design engineers, when developing a data transmission system that employs pulse shaping.

To satisfy the ever increasing demands for higher data rates as well as to allow more users to simultaneously access the network, interest has peaked in what has come to be known as WCDMA The WCDMA has emerged as the most widely adopted $3 \mathrm{G}$ air interface and its specification has been created in 3GPP .In this system the user information bits are spread over much wider bandwidth by multiplying the user data bits with quasi random bits called as chips derived from CDMA spreading codes.

In order to support very high bit rates (up to $2 \mathrm{Mbps}$ ) the use of variable spreading factor and multimode connection is supported. The chip rate of $3.84 \mathrm{Mcps} / \mathrm{sec}$ is used to lead a carrier bandwidth of 5Mhz.WCDMA also supports high user data rates and increased multipath diversity [33].Here each user is allocated the frames of $10 \mathrm{~ms}$ duration during which the user data is kept constant though data capacity among users can change from frame to frame. The preferred technology for third-generation systems depends on technical, political, and business factors. Technical factors include issues such as provision of required data rates, and performance. Political factors involve reaching agreement between standards bodies and taking into account the different starting points of different countries and regions. On one hand, the investments into the existing systems motivate a backward compatibility approach. On the other, new business opportunities or the possibility of changing the current situation might motivate a new approach.

The past, present, and future activities of CDMA are presented in the next section. The following section explains the basic concepts and elements of CDMA. Then the IS-95 air interface is introduced according to the new IS-95 standard, followed by a brief discussion of air interface technologies for third-generation, with a short description of TD-CDMA. Wideband CDMA schemes are then discussed in great length and conclusions are given in the final section.

To satisfy the ever increasing demands for higher data rates as well as to allow more users to simultaneously access the network, interest has peaked in what has come to be known as wideband code division multiple access (WCDMA).The basic characteristics of WCDMA waveforms that make them attractive for high data rate transmissions are their advantages over other wireless systems. It emphasizes that how the choice of spread bandwidth affects the bit error rate of system.

\section{IV.WCDMA SCHEME}

The WCDMA communication link proposed in this section is shown in Figure 1.0. The performance in terms of the Bit Error Rate can be examined for different values of Group Delay D of the pulse shaping filter against a sinusoidal interference. A simulink model based on the Matlab 7.3 version will provide the output. The information signal in wideband CDMA system is generated by Bernoulli Binary Generator and the PN sequence is used for spreading the signal at $5 \mathrm{MHz}$ bandwidth. The signal is passed from different parameters block as shown in figure 1.0 and at the end BER is calculated by comparing the transmitted data and received data. On the basis of above block diagram, a simulation model has been developed by using Matlab Simulink Library as shown in figure 1.0

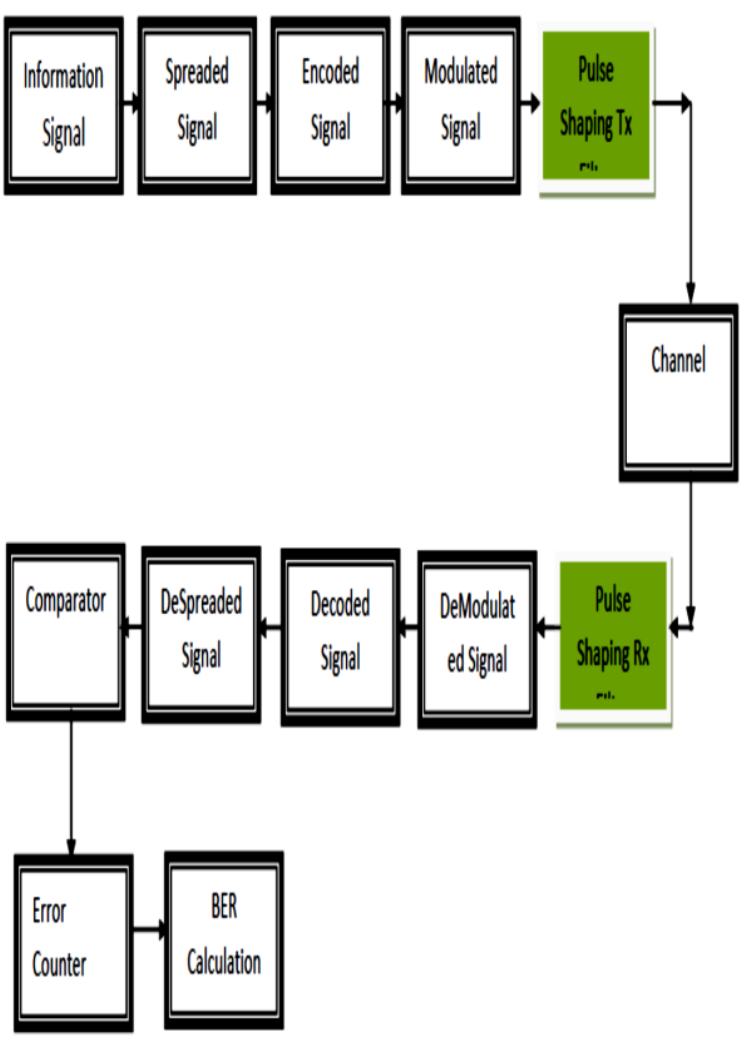

Fig.1.0 WCDMA System

\section{V.RESULT}

I have investigated our WCDMA simulink model at different AWGN channel noise SNR. The values of SNR are preferred as 510 and $15 \mathrm{~dB}$.At these SNR the BER performance is calculated for different values of roll off factor and delay of our root raise cosine filters.

Plots at $\mathrm{SNR}=5 \mathrm{~dB}$ :

At SNR equal to $5 \mathrm{~dB}$ delay of RRC filter roll of factor is varied from 0.102 to 0.114 as shown in figure 2.0.It has been observed that the $3 \mathrm{D}$ plot of BER with respect to delay (D) and Roll off factor (B) the minimum BER is obtained at $\mathrm{D}=6$ for $\mathrm{B}=0.103$. 
Vol. 4, Issue 8, August 2015

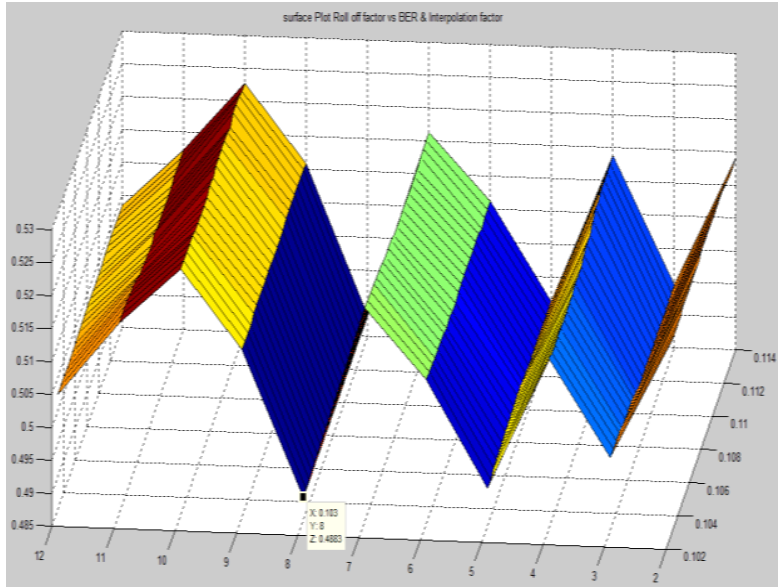

Fig. 2 .0 BER vs Delay and Roll off factor plot at $\mathrm{SNR}=5 \mathrm{~dB}$

In above figure $\mathrm{Y}$ indicates delay and $\mathrm{x}$ indicates roll off factor and the global minimum point exist at $\mathrm{D}(\mathrm{i} . \mathrm{e} \mathrm{Y})=6$ and $\mathrm{B}(\mathrm{i} . \mathrm{e})=0.103$ the value of BER is demonstrated as $\mathrm{Z}$ axis and at this minimum point the BER (i.e $\mathrm{Z}$ ) $=0.4883$.

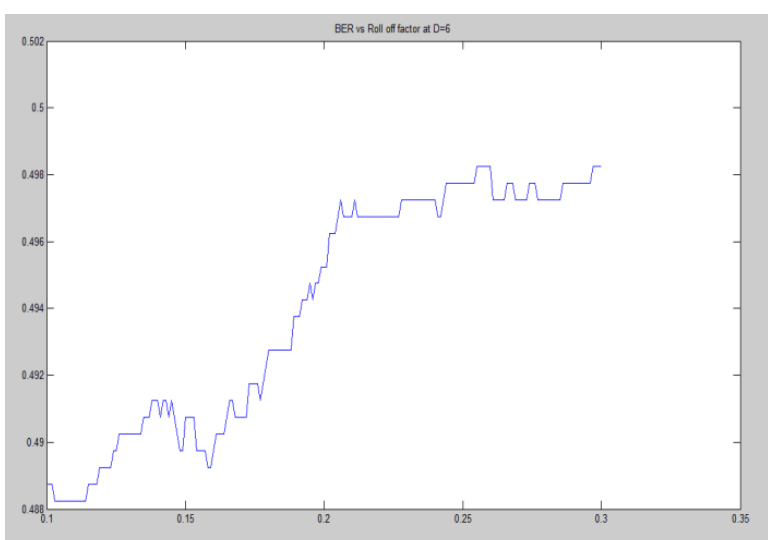

Fig.2.1 BER vs. Roll off (B) plot to track range of B for minimum $\mathrm{BE}$

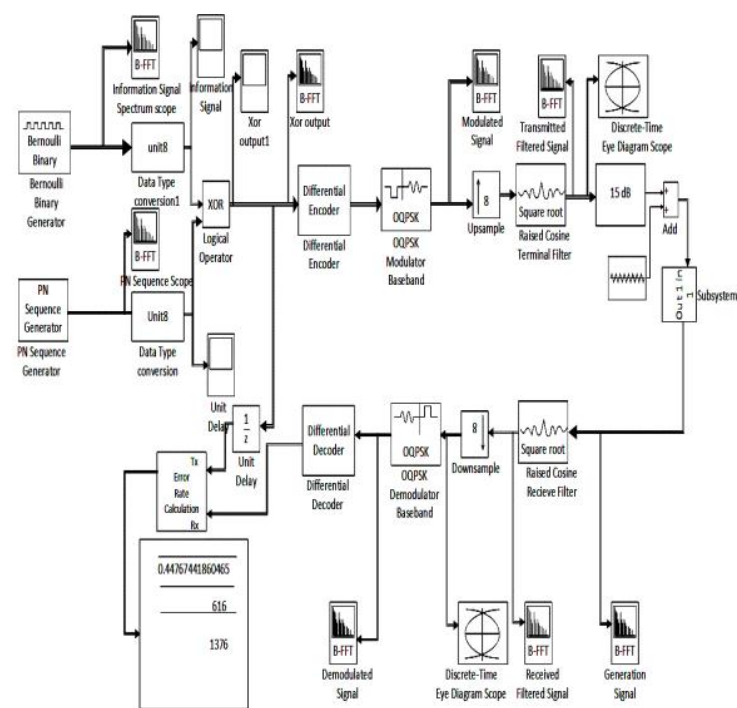

Fig. 4.0 WCDMA based Simulation Model developed for Square Root Raised Cosine Pulse reduces to 0.488 for $\mathrm{B}=0.103$ to 0.114 after that for higher $\mathrm{B}$ the value of BER is increasing.

\section{Plots for $\mathrm{SNR}=10$}

At SNR equal to $10 \mathrm{~dB}$ the delay of RRC filter is varied from 1 to 10 and roll of factor is varied from 0.102 to 0.114 as shown in figure below. It has been observed that the 3-D plot of BER with respect to delay (D) and Roll off factor (B) the minimum $B E R$ is obtained at $D=6$ for $\mathrm{B}=0.103$.

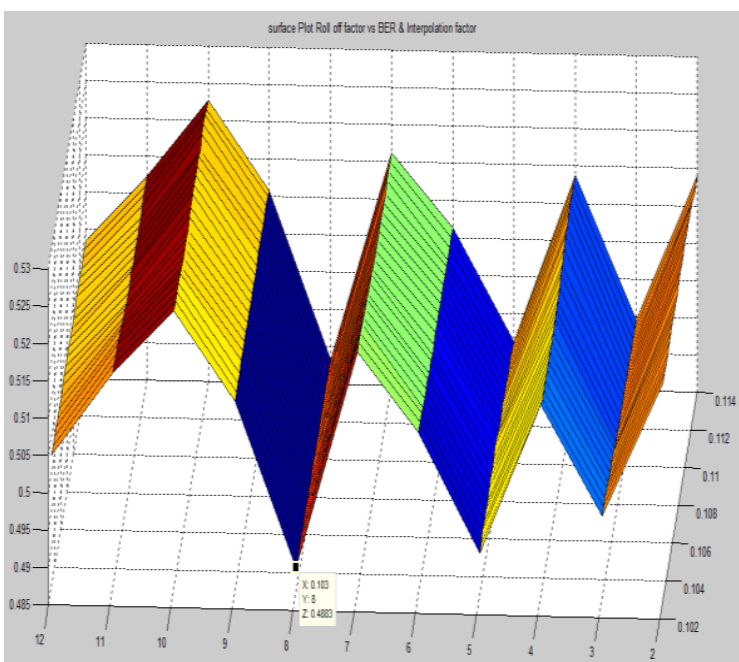

Fig. 3.0 BER vs Delay and Roll off factor plot at $\mathrm{SNR}=10 \mathrm{~dB}$

At optimum delay $\mathrm{D}=6$ for $\mathrm{RRC}$ filter we again varied the roll off factor B to check that which value of B supports for minimum $B E R$ at $D=6$. The same observation as we obtained for $5 \mathrm{~dB}$ are repeated as shown in figure 1.0 and 1.1

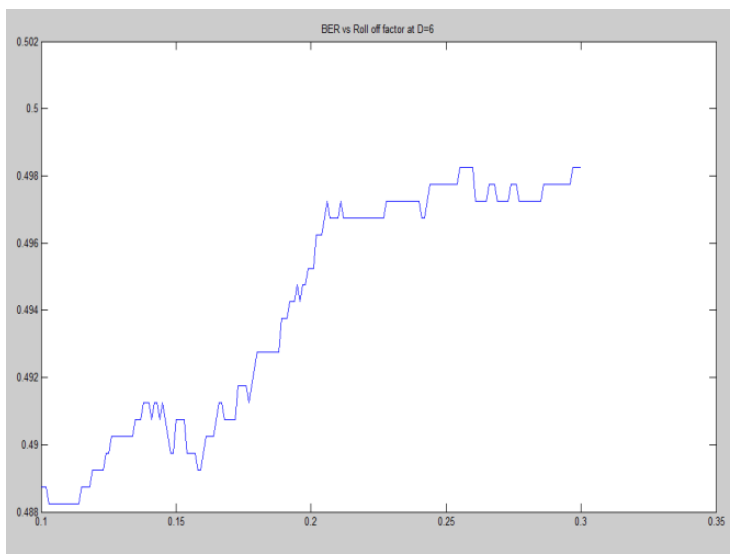

Fig. 3.1 BER vs. Roll off (B) plot to track range of B for minimum BER.

Similar studies are also performed for higher SNR at $15 \mathrm{~dB}$.As it is evident that foe higher SNR noise is reduced due to this error rate decreases. We can see in figure 5.3 that minimum BER is obtained equal to 0.4455 at delay $\mathrm{D}=6$ and $\mathrm{B}=0.07$. It indicates that for delay $\mathrm{D}=6 \mathrm{BER}$ is minimum and it reduces with SNR We have also analyzed the performance of pulse shaping effect in WCDMA model using eye diagram scatter plot and spectrum scope. We have used spectrum scope plot for showing the magnitude spectrum of output of or before QPSK. Discrete time eye diagram and scatter plot after QPSK modulation at transmitter side. 


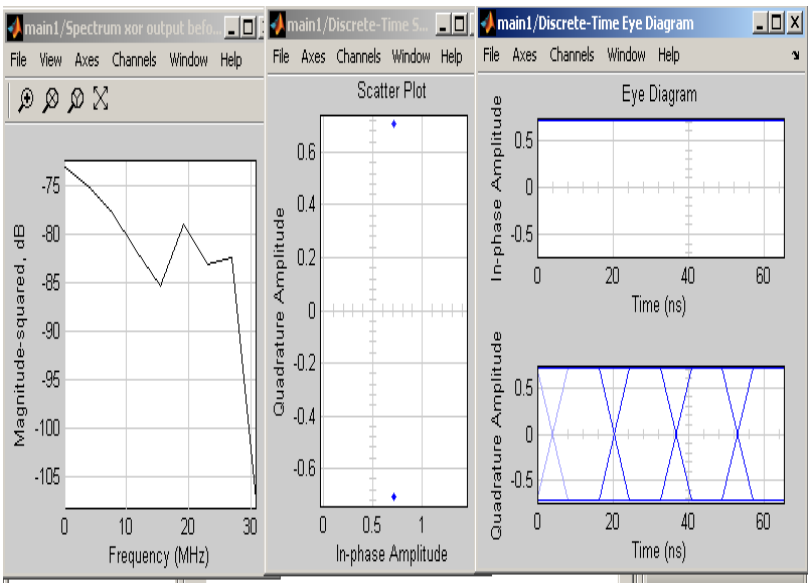

Fig.5.0 Response of transmitter side at spectrum scope (left) scatter plot (mid) and eye diagram (right)

In figure 5.0and 5.1 we have shown six scope out of which 3 belongs to transmitter end and remaining 3 at receiver end after distortion created by transmitter side and AWGN channel. Figure 5.0 to 5.3 represents the response of WCDMA under the effect of pulse shaping filter at different value of delay $\mathrm{D}$ for root raise cosine filter.

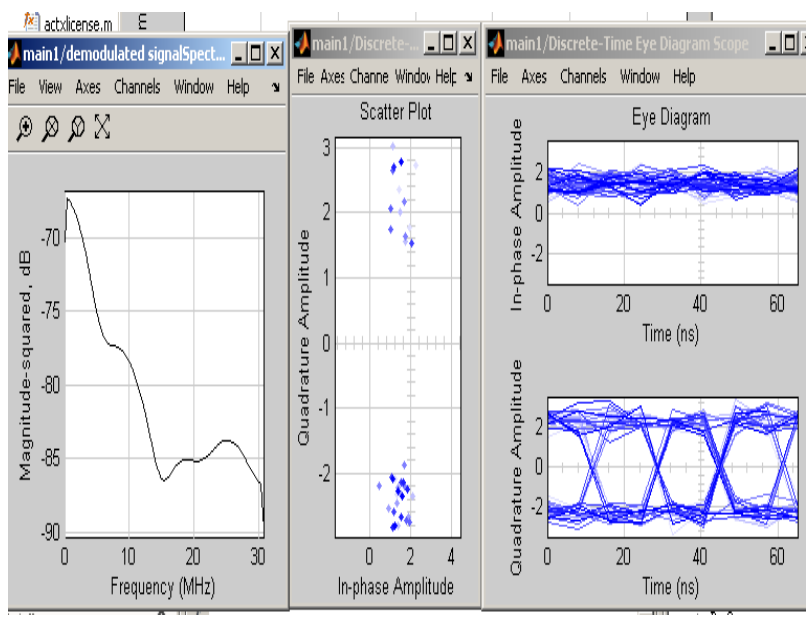

Fig.5.1Response at receiver side at $\mathrm{D}=2 \mathrm{SNR}=5 \mathrm{~dB}$ $\mathrm{BER}=0.4921$ (left) scatter plot (mid) and eye diagram (right).

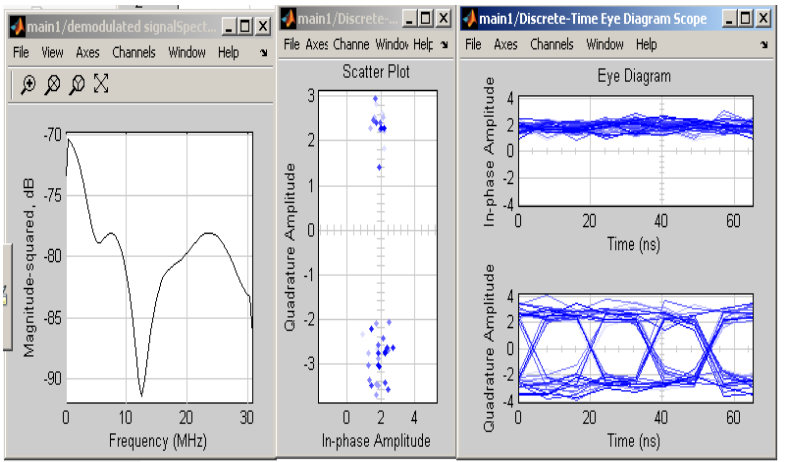

Fig.5.2 Response at receiver side at $\mathrm{D}=4 \mathrm{SNR}=5 \mathrm{~dB}$ $\mathrm{BER}=0.5055$ (left) scatter plot (mid) and eye diagram (right).
Here SNR of AWGN channel is kept constant at $5 \mathrm{~dB}$ i.e. noise is large and roll of factor is kept at 0.103. As the D is rising we noted down the value of BER between transmitted and received signal.

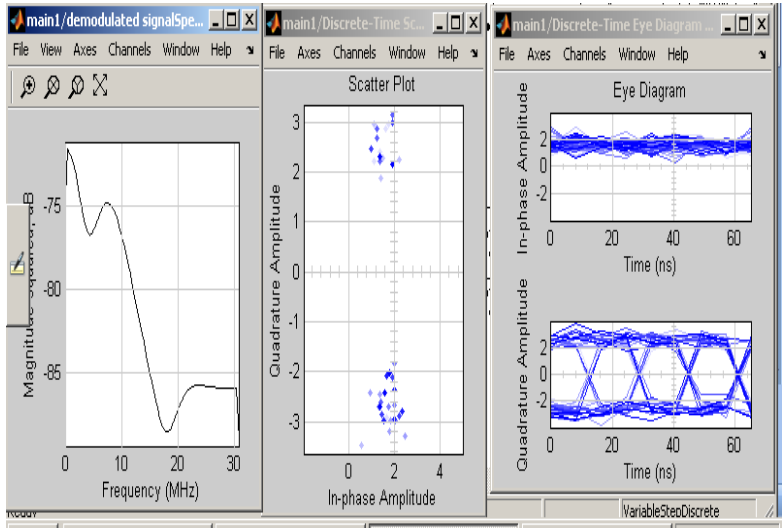

Fig.5.3 Response at receiver side at $\mathrm{D}=6 \mathrm{SNR}=5 \mathrm{~dB}$ $\mathrm{BER}=0.4884$ (left) scatter plot (mid) and eye diagram (right).

The error can be visualized by observing the scatter plot and eye diagram. (Fig.5.1 to 5.3) Scatter plot for modulation case are showing only two points but after receiving and prior to demodulation these signals are scattered in neighborhood to reference transmitted points. For $\mathrm{D}=6$ scattering is minimum. Similarly in all cases eye diagram is also get distorted in both quadrature and in phase amplitudes.

\section{CONCLUSION}

\begin{tabular}{|l|l|l|l|}
\hline Delay (D) & $\begin{array}{l}\text { BER at } \\
\text { SNR 5dB }\end{array}$ & $\begin{array}{l}\text { BER at } \\
\text { SNR 15dB }\end{array}$ & $\begin{array}{l}\text { BER at } \\
\text { SNR 25dB }\end{array}$ \\
\hline 2 & 0.4911 & 0.4912 & 0.492 \\
\hline 4 & 0.5055 & 0.5055 & 0.5055 \\
\hline 6 & 0.4884 & 0.4559 & 0.4214 \\
\hline 8 & 0.4947 & 0.4974 & 0.4974 \\
\hline
\end{tabular}

Table1.0 Delay vs. BER at different SNR

The effect of variation of group delay D i.e. number of symbols spanned by impulse response is studied at different value of roll off factor $B$ at well as at fix value of interpolation $\mathrm{M}=8$. The study has impact on analysis \& simulation of pulse shaping families in WCDMA based wireless communication system. It is also observed that BER decreases as the group delay is increased from 2 to 4 and then from 4 to 6 . The BER is found to increase as the value of group delay $D$ is varied from 6 to 8 . Hence the group delay should be controlled at $\mathrm{D}=6$ by $\mathrm{RF}$ design engineer.The range of roll off factor for keeping minimum BER is found to be 0.103 to 0.114 . Hence the optimum value of $\mathrm{D}=6$ and $\mathrm{B}=0.12032$ to 02.114 should taken for the design of the WCDMA system. The study will be useful to improve the performance of WCDMA based network by using the modified and improved design of square root raised cosine pulse shaping filter. Design of new type of filter of higher or different order will be useful 
to get better root raised cosine approximation thereby improving the performance parameters like increased Capacity, reduced BER, better $\mathrm{S} / \mathrm{N}$ ratio, and Reduced ISI (noise) as a consequence of pulse shaping. The future work will involve the incorporation of interpolation factor for trade off between $\mathrm{D}$ and $\mathrm{M}$ at fix roll off factor as well as study of parameters of pulse shaping filter on the bit error rate performance analysis for WCDMA based wireless communication. We can also apply advanced optimization techniques like GA and PSO to find and optimum set of values of delay roll off factor and interpolation factor for achieving an objective of minimum BER and higher transmission capacity.

\section{REFERENCES}

[1]. Fred Harris, on the design of preequalized Square root raised cosine matched filters for DSP based digital receivers" IEEE -10586393/93, pp-1291-95, (1993).

[2]. Jun He Colin, "Performance of SRC filtered ODQPSK in mobile Radio communications", IEEE 07803-1266-x/93, pp-668-671, (1993).

[3]. Xiang Gen Xia,"A Family of Pulse Shaping Filters with ISI Free Matched and Unmatched Filter Properties" IEEE Transactions on Communications, VOL 45, NO 10,(Oct 1997).

[4]. Ye Li KJ, Ray Liu,"Signal Processing for Wireless communication" IEEE journal on selected areas in communication, vol-16, no.8, pp1337-1339, (Oct1998)

[5]. A.V Kisel"An extension of pulse shaping filter theory" IEEE transactions on communication, vol47 no5, pp645-47,(1999)

[6]. N S Alagha and P Kabal,"Generalized raised cosine filters, "IEEE transaction on communication ,vol 47, no7,pp989-997,(1999) 